\title{
MECHANISM DESIGN, DYNAMICS MODELLING AND EXPERIMENTS OF BIONIC UNDULATING FINS
}

\author{
Haibin Xie, ${ }^{*}$ Han Zhou, ${ }^{*}$ Lincheng Shen,${ }^{*}$ and Dong Yin*
}

\begin{abstract}
The undulating fin propulsion is inspired by fish using Median and/or Paired Fin (MPF) mode. This mode provides advantages of the vector thrust production and small disturbance to the ambient flow field, and also it could be applied on underwater robots conveniently. Two bionic undulating fins are designed to imitate the structure and function of undulating fin of aquatic animals, which are fixed-waveform mode and independently-driven mode. The active deformation of bionic undulating fins is described by a kinematic model. Base on the kinematic model, a simplified computational model is derived theoretically to analyse the dynamics of the bionic propulsor. The dynamic model considers six components of forces and moments. The dynamics performance related to the geometric parameters, undulating parameters as well as the carrier velocity are further discussed through simulation. Furthermore, the above analytic method is verified through the thrust/moment and velocity test using the bionic propulsor.
\end{abstract}

\section{Key Words}

Undulating fins, bionic propulsor, dynamics modelling, mechanism design

\section{Introduction}

Fish locomotion can be classified into body and/or caudal fin (BCF) and median and/or paired fin (MPF) [1], [2]. As shown in Fig. 1, Amiiform, Gymnotiform, Balistiform, Rajiform are regarded as MPF mode. These fishes can generate thrust using undulating fins while their bodies keep rigid and straight. The thrust magnitude and direction is controlled by changing waveform, wavelength, frequency, amplitude and wave travelling direction [3], [4]. Various bionic prototypes are designed to imitate fishlike locomotion and used for mechanism study [5]-[21].

The propulsion mode of undulating fin represents some remarkable advantages, such as producing vector thrust

\footnotetext{
* College of Mechatronics and Automation, National University of Defense Technology, Changsha, 410073, P.R. China; email: xhb2575_sx@sina.com, ivyhzhou@163.com, lcshen@nudt.edu.cn, yindong@nudt.edu.cn

Recommended by Prof. Chaomin Luo
}

(DOI: 10.2316/Journal.206.2016.2.206-4665)

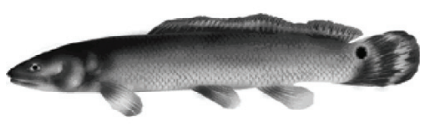

(a)

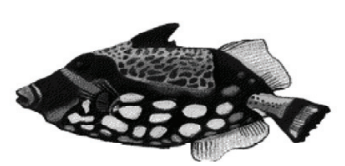

(c)

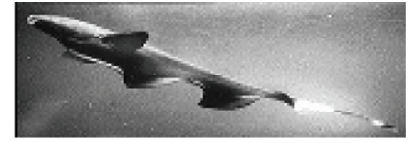

(b)

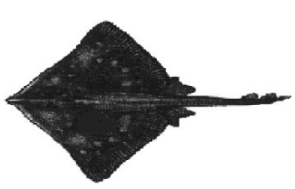

(d)
Figure 1. Fishes using undulating MPF mode [8]: (a) Amiiform; (b) Gymnotiform; (c) Balistiform; and (d) Rajiform.

and smaller disturbance to ambient flow field. The propulsion mode provides a bionic inspiration for underwater robots. Many research institutes have been developed the research on the undulating fin [4], [9-19], and the dynamics mechanism and prototype development of the undulating fin are hot topics in this field [5], [20]-[23].

In this paper, two different bionic propulsors are introduced which imitate the structure and function of the undulating fin. The kinematics and dynamics modelling is developed for the bionic undulating propulsion. Those theoretical results are further tested by proceeding experiments of the bionic fin.

\section{Bionic Propulsor Inspired by Undulating Fish Fin}

We have been studying the propulsion mechanism of the undulating fin since 2003. The research has mainly focused on the kinematics and morphology observation of bionic objects, dynamics modelling and analysis, computational fluid dynamics simulation, etc. [4], [8], [18]. A bionic fin was designed for the performance test of the bionic undulating propulsion and control system of bionic underwater robots in 2003. The bionic propulsor used a fixedwaveform scheme to realize rapid function verification, of which the transmission mechanism and control system has the minimal complexity. Based on this bionic undulating 


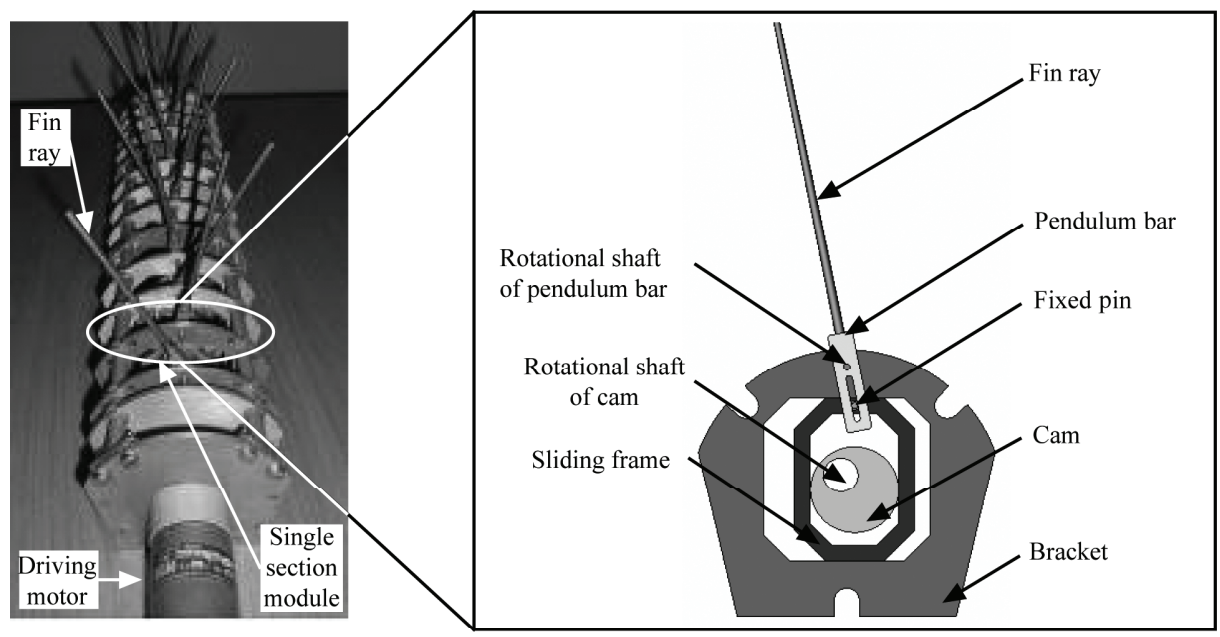

(a)

(b)

Figure 2. Structure of the fixed-waveform bionic undulating fin: (a) the actuating mechanism and (b) the single section module.

fin, a prototype of underwater vehicles was also developed. In 2005, we further developed a new bionic undulating fin using the independently driven scheme. Each fin ray of the new bionic fin was actuated by independently controllable digital servomotor. Therefore this bionic fin presented flexibility on imitating multiple wave modes. The fixedwaveform and independently driven bionic fins are next described in detail, respectively.

\subsection{Fixed-Waveform Bionic Undulating Fin}

The fixed-waveform bionic fin imitates fish fins in structure and produces propulsive travelling waves. It is formed of a flexible fin surface and an actuating mechanism. The fin surface includes several fin rays and a flexible membrane, which are made by spring steel and elastic latex materials, respectively. The fin rays are imbedded in the flexible membrane. The fin surface is designed not only considering the flexibility and stretchability, but also minimizing its average inner stress under any motion parameters. All of the fin rays have uniform length. All of the fin rays are driven by the actuating mechanism, to oscillate in accordance with the fixed phase relationship. As a result, the travelling wave generates on the fin surface, which is analogous to some wave mode of the fish undulating fin. The undulating frequency is the only controllable parameter for the fixed-waveform bionic fin and other wave parameters are solidified in the mechanism. The actuating mechanism consists of 13 sets of section modules, as shown in Fig. 2(a). The single section module is a minimal device to realize the oscillation of each fin ray, which consists of a cam, a sliding frame, a bracket and a pendulum bar, as shown in Fig. 2(b). The single section module is actuated by motor and transmitted by cam. The mechanism changes the motor's rotating motion into the fin ray's periodic oscillating motion. All modules are connected by one long shaft, which is driven by one motor. The phase relationship of the fin rays depends on the mounting phase of the cam relative to the long shaft. The rotational direction of the long shaft decides the wave propagation direction. The wave frequency is in proportion to the rotation speed of the long shaft. Accordingly, the wave velocity and travelling direction are able to be adjusted by the motor's rotation rate and direction. It has compact structure and simple control system for this scheme. However, the disadvantage is the loss of control agility.

- Prototype of bionic underwater vehicle

The prototype of bionic underwater vehicle adopts the fixed-waveform bionic fin as propulsor, which is used to supply vector thrust for the prototype and realize the forward and backward propulsion as well as braking control. In addition, one criss-cross tail vane with two degrees of freedom is used as the steering control module, and one barycentre adjustment module is used as the pitching and balance control module.

a. Steering control module consists of one criss-cross tail vane and two driving servomotors used to respectively control the level and vertical degree of freedom of the tail vane. During the sailing course, adjusting the angle of the vertical plane (level degree of freedom) of the tail vane can supply yawing moment to realize the steering control, and controlling the angle of the level plane (vertical degree of freedom) of the tail vane can also supply additional pitching moment to aid the pitching and balance control.

b. Pitching and balance control module is made up of a barycentre adjustment module and its controldriven system. The module can produce pitching moment through adjusting the axial displacement of the barycentre, and accordingly realize the dynamic balance control along the axial direction of the bionic underwater vehicle and pitching attitude control. The test model can also realize self-stability of the rolling degree of freedom through downward collocating the barycentre of the carrier.

With the collaborative operation of these three parts, the prototype can control the thrust magnitude and direction, axial displacement of the barycentre, as well as 


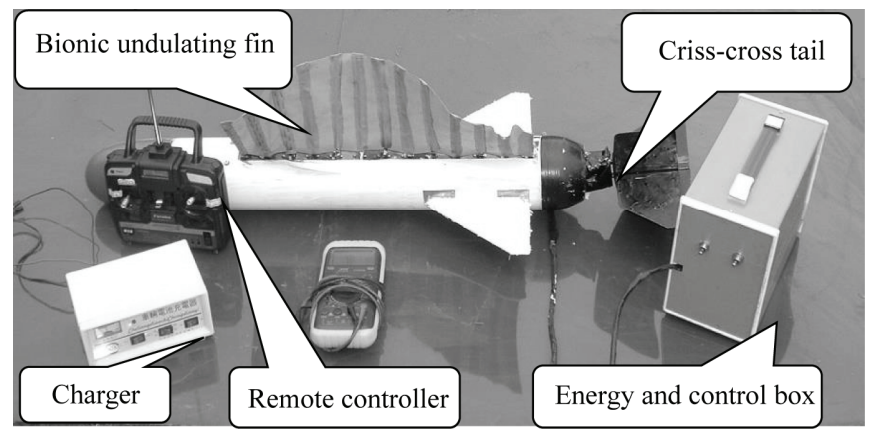

Figure 3. Prototype of bionic underwater vehicles.

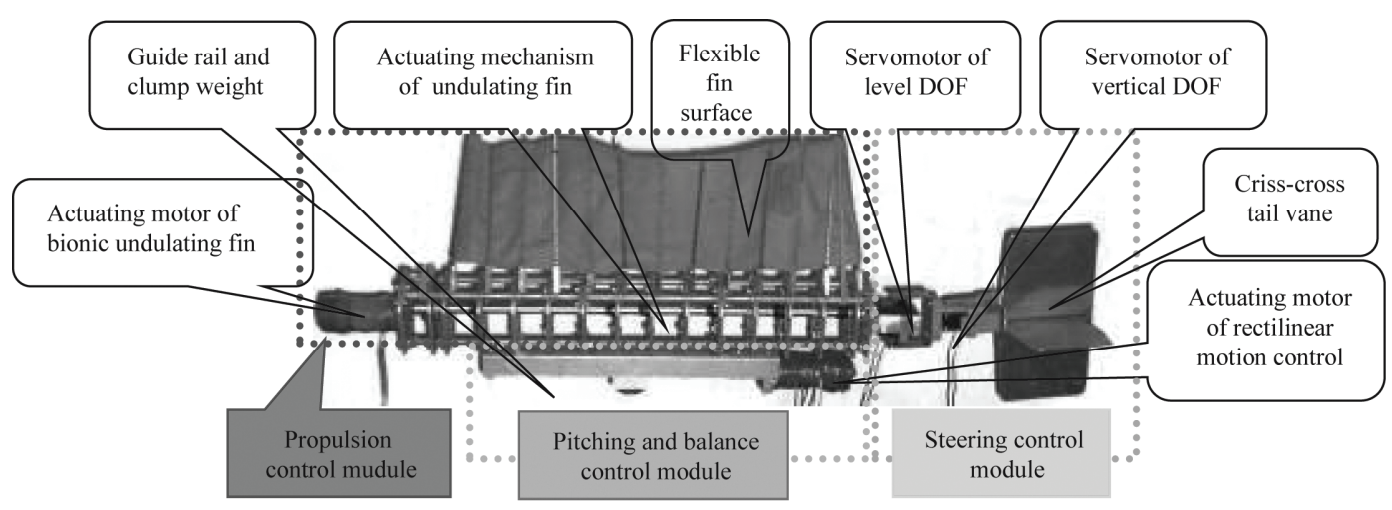

Figure 4. Structure of the bionic underwater vehicle.

angle and direction of the criss-cross tail vane, respectively. Therefore, the prototype not only has the capabilities of realizing the sailing stability control as well as the following basic motion modes, such as advancing, backing, braking, turning and pitching, but also can realize more complex three-dimensional motion through the combination control of the above-mentioned basic motion modes. We have carried on a series of integration tests and swimming trials on the prototype. The bionic undulating fin can swim both forward and backward, and complete the basic motions such as turning and pitching manoeuvre. The disturbance to the surrounding flow field and the noise is small. The prototype of bionic underwater vehicle and its system construction are shown in Figs. 3 and 4, respectively.

\subsection{Independently Driven Bionic Undulating Fin}

There is a great limitation for the fixed-waveform bionic fin to reproduce the fish fin's flexible motion. Another bionic undulating fin is developed, which has 17 fin rays. The structure is also designed as motor actuates and gear transmits. Each fin ray is actuated and controlled independently, so each fin ray could oscillate as the desired motion while its oscillating angle can be real-time adjusted. Therefore, the independently driven bionic undulating fin allows greater flexibility on imitating the propulsive wave motion. The normal sinusoid wave can be presented on the fin surface with 1-4 wave numbers, in light of the Nyquist sampling theorem. Figure 5 shows the structure of independently driven bionic fin, which includes the fin's actuating mechanism and a single fin ray's swing mechanism.

For the fixed-waveform mode, it is a simple and efficient way to control the complicated structure, which brings about the friction, resulting in low efficiency and reliability. This device is mainly used for the qualitative study of wave propulsion performance. For the independently driven mode, every single fin ray is actuated by an independent servo motor and flexible control is realized. This mode can be used for simulating a variety of propulsion wave modes, and accurately studying the quantitative and qualitative effects of undulating parameters. The control system is more complex than that of fixed-waveform mode. In addition, the motor reliability will infect the performance of wave motion.

\section{Kinematics Analysis of Bionic Undulating Fin}

\subsection{Kinematics Modelling}

The fin surface coordinate system is defined as shown in Fig. 6 [4]. The baseline of the fin is considered as a fixed straight line. Each fin ray oscillates about a fixed point in a specific plane. The fin rays have no flexibility. The spatial motion surface model of the bionic undulating fin can be established as follows [4], [18]:

$$
\left\{\begin{array}{l}
x(s, l, t)=s \\
y(s, l, t)=l \cos [\theta(s, t)] \quad s \in[0, L], \quad l \in\left[0, L_{q}\right] \\
z(s, l, t)=l \sin [\theta(s, t)]
\end{array}\right.
$$




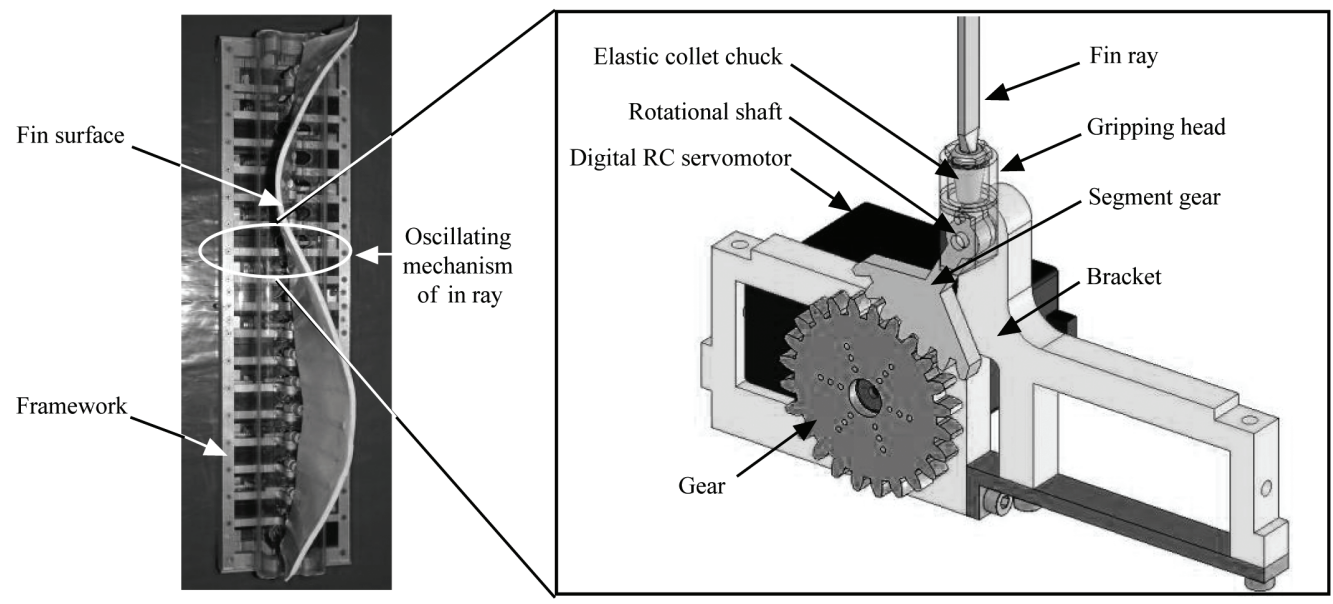

(a)

(b)

Figure 5. The structure of independently driven bionic undulating fin: (a) the actuating mechanism and (b) oscillating mechanism of the fin ray.

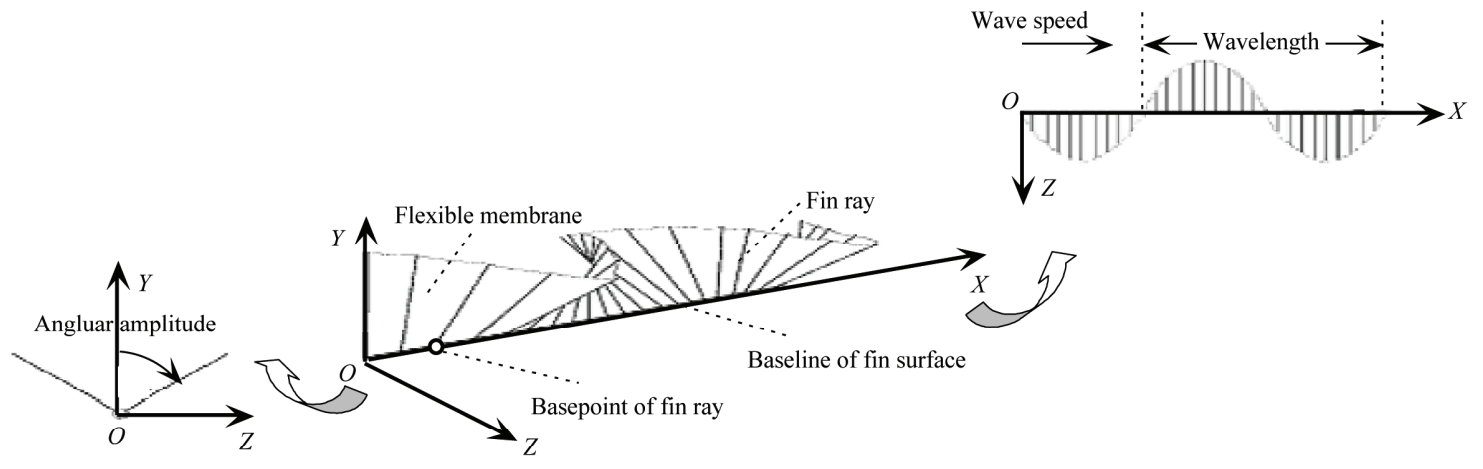

Figure 6. The fin surface coordinate system.

where $t$ is the time; $s$ is the coordinate along the baseline of the fin; $l$ is the coordinate along fin ray; $L$ is the length of the whole fin; $L q$ is the length of a fin ray and $\theta(s, t)$ is the oscillating angle, which describes the motion of fin ray functionally.

Each fin ray oscillates periodically around its respective basic point. Because the structure and driving system is same for all the fin rays, it is supposed that all fin rays complies with the uniform motional law. Therefore, we could define all the fin rays' motion obey an identical periodic function, and parameters' values are different for each fin ray. The periodical motion law $\theta(s, t)$ could thus be expressed as:

$$
\theta(s, t)=A(s) F(s, t)+\theta q(s)
$$

where $A(s)$ is the oscillating amplitude of the fin ray at point $s ; F(\cdot)$ is a periodic function of $\varepsilon(t)$, whose period is $2 \pi$ and the value interval is $[-1,1]$, i.e., $F[\varepsilon(t)]=F[\varepsilon(t) \pm 2 \pi] ; \varepsilon(t)$ is a linear function of time $t$, which is $\varepsilon(t)=2 \pi f t+\varepsilon_{0}(s) ; \varepsilon_{0}(s)$ is the initial motion phase of the fin ray at points; $f$ is the undulating frequency and $\theta q(s)$ is the deviation angle of the fin ray at point $s$.

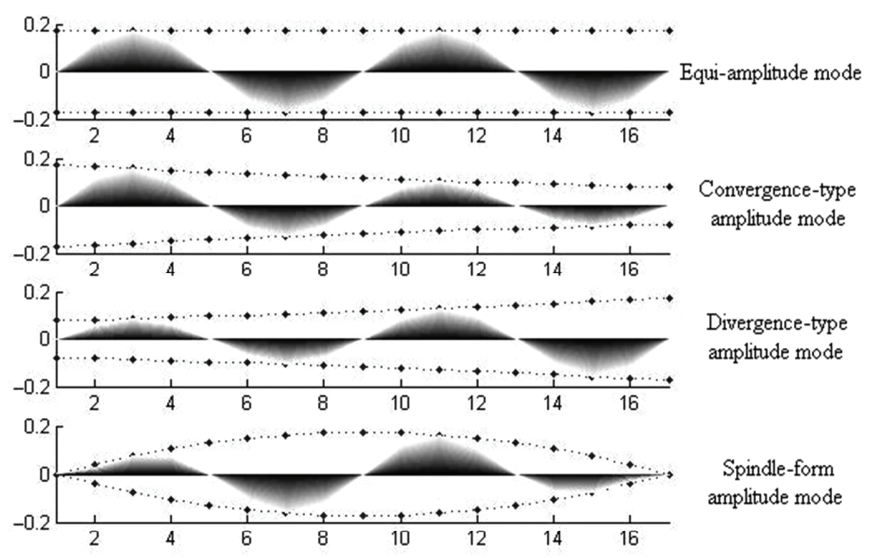

Figure 7. Wave amplitude of the undulating fin.

\subsection{Analysis of the Kinematics Model}

Based on the kinematics model, $F(\cdot), f, A(s), \varepsilon_{0}(s)$ and $\theta q(s)$ could completely determine the propulsive wave mode, if the fin surface length $L$, fin ray length $L q$, as well as the number and spacing of fin rays have already been set. $F(\cdot)$ describes periodical oscillating motion of 


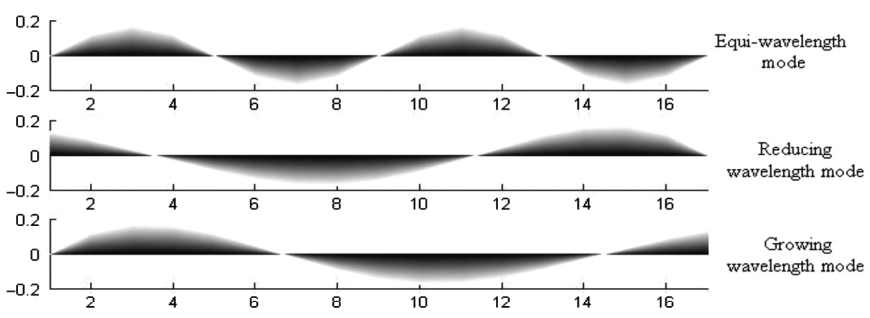

Figure 8. Wavelength of the undulating fin.

the fin ray. $f$ describes the frequency of the propulsive wave, whose sign (positive or negative) also denotes the direction of wave travelling. $A(s)$ describes the amplitude of propulsive wave. If $L q$ has been determined, $A(s)$ is responsible for undulation modes of variable amplitudes, e.g., divergence-type, convergence-type and spindle-form. $\varepsilon_{0}(s)$ describes the phase relation of fin rays. $\varepsilon_{0}(s) / 2 \pi f$ represents the time delay of the fin rays' motion. If $\varepsilon_{0}(s)$ is a constant, all fin rays oscillate synchronously, i.e., standing wave mode. If $\varepsilon_{0}(s)$ is a linear function, e.g., $\varepsilon_{0}(s)=(2 \pi / \lambda) s+\varepsilon_{0}(0)$, the propulsion wave is generated with wavelength $\lambda$, i.e., equi-wavelength mode. $\varepsilon_{0}(s)$ can describe the undulating mode of variable wavelengths as well. Moreover, the initial phase reflects the time-varying course of the wavelength if expressed as $\varepsilon_{0}(s, t) . \theta q(s)$ is the degree of each fin ray' motion symmetry axis deviating from plane $O x y$, which allows the fin motion adapts to the varied surrounding flow fields and optimizes its propulsion performance.

These analyses will be further verified by simulation. For the independently driven bionic fin, there are 17 rigid fin rays in equal distribution. All the fin rays have unit length; $F(\cdot)$ is a sine function. $\theta(s, t)$ is expressed as follows:

$$
\theta(s, t)=A(s) \sin \left[2 \pi f t+\varepsilon_{0}(s)\right]+\theta q(s)
$$

Figures 7-9 show the wave modes of simulation, where $A(s), \varepsilon_{0}(s)$ and $\theta q(s)$ decide different undulating modes of propulsion waves. Figures 7 and 8 display the projection of fin surface on plane $O x z$, where the horizontal ordinate uses serial number $s$ of the fin ray, $s=1,2, \ldots, 17$. Figure 9

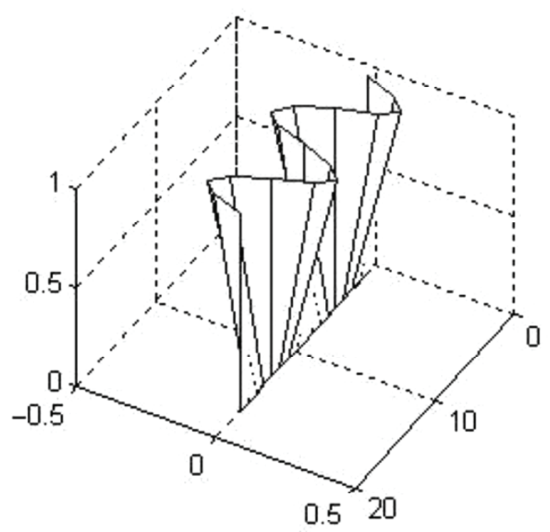

shows the 3D fin surface, in which $x$-coordinate is serial number $s, y$ - and $z$-coordinates are dimensionless number.

Herein, the undulating mode is considered with uniform amplitude, wavelength and frequency. There is no lateral deviation and it is sinusoidal oscillating for our designed bionic fin. Then, the angle of fin ray $\theta(s, t)$ is represented as:

$$
\theta(s, t)=\theta_{\max } \sin \left[2 \pi f t+\varepsilon_{0}(s)\right]
$$

where $\theta_{\max }$ is the maximum oscillating angle, and the lateral deviation $\theta q(s)=0 . \varepsilon_{0}(s)$ describes the uniform wavelength mode, which is set as $\varepsilon_{0}(s)=\varepsilon_{01}-2 \pi s / \lambda$, where $\varepsilon_{01}$ is the initial phase of the first fin ray. The kinematics modelling of the bionic fins is illustrated in Fig. 10.

\section{Dynamics Modelling of Bionic Undulating Fin}

\subsection{Dynamics Modelling}

The hydrodynamic mechanism of the undulating fin propulsion is transient and unsteady. The fin's active and passive motion has the coupling relationship with the surrounding flow field. The study on hydrodynamics effect and energy consumption has remained an ongoing work [24]-[27]. Although the fluid drag model does not take the influences of fluid motion into consideration, it is still effective for qualitatively understanding the dynamics characteristics of the undulating fin [11]. The fluid drag model assumes that [4], [11]: (1) during the underwater locomotion, hydrodynamic forces acting on any surface element are divided into normal stress and shear stress; (2) the normal stress and shear stress are related to the instantaneous velocity, as presented in the following:

$$
\begin{aligned}
& \vec{f}_{n}=\vec{F}_{n} / S=-\frac{1}{2} \rho C s\left\|\vec{v}_{n}\right\|^{2} \vec{v}_{n_{0}} \\
& \vec{f}_{\tau}=\vec{F}_{\tau} / S=-\frac{1}{2} \rho C \tau\left\|\vec{v}_{\tau}\right\|^{2} \vec{v}_{\tau_{0}}
\end{aligned}
$$

where $\vec{f}_{n}$ and $\vec{f}_{\tau}$ are the stress vectors acting perpendicularly and in parallel with the propulsive element of the fin surface, respectively. $\vec{F}_{n}$ and $\vec{F}_{\tau}$ are the corresponding

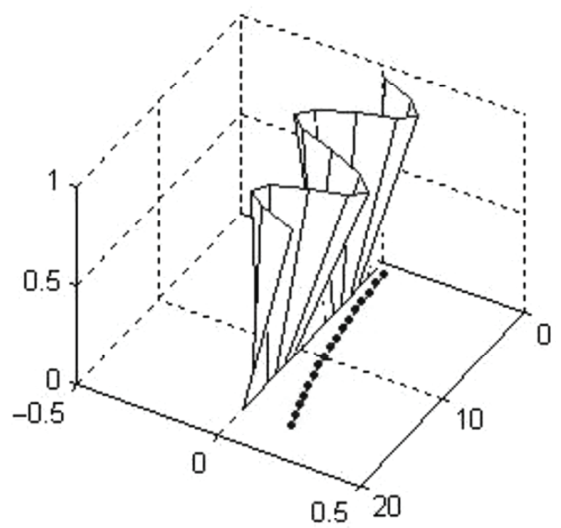

Figure 9. Lateral deflection of the undulating fin. 

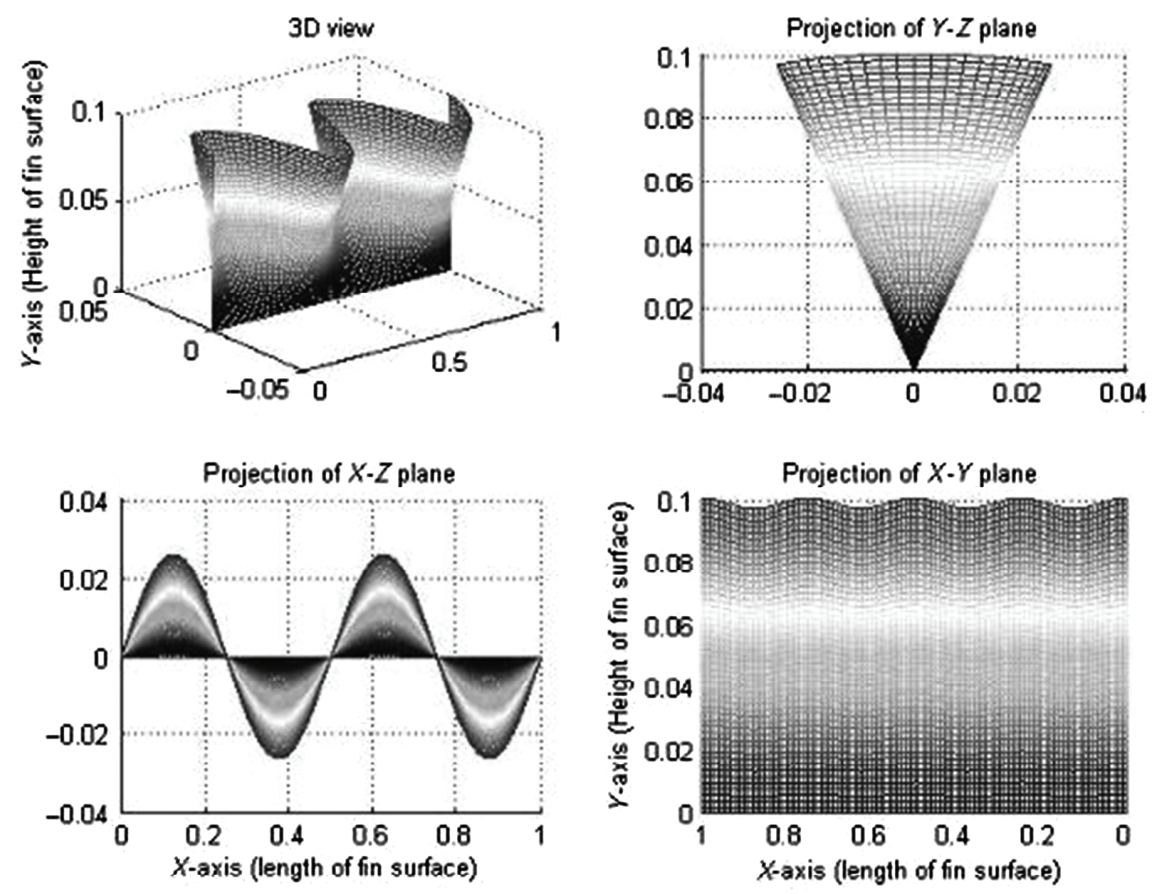

Figure 10. Kinematics modelling for the bionic undulating fins.

force vectors. $\vec{v}_{n}$ and $\vec{v}_{\tau}$ are the instantaneous normal and tangential velocities of the surface element relative to the fluid, respectively. $\vec{v}_{n_{0}}$ and $\vec{v}_{\tau_{0}}$ are the corresponding unit vectors. $S$ is the area of the surface element. $C s$ and $C \tau$ are the drag coefficients which is shape-dependent. $\rho$ is the density of the surrounding fluid.

Furthermore, there are two more assumptions for our problem: (1) The Reynolds number $\operatorname{Re}=v_{b} L / \nu$ exists in $10^{6}$ orders of the magnitude, where $v_{b}=\omega \lambda / 2 \pi$ is the phase speed of the propulsion wave, and $\nu$ is the viscosity coefficient of fluid. The undulating propulsion belongs to the hydrodynamic problem of high Reynolds number. Therefore it is regarded as non-viscous fluid and then the viscous resistance is negligible. (2) The fin length and wavelength satisfy the equation $L=n \lambda$, where $n$ is an integer. Then the undulating fin surface presents periodicity and symmetry.

The above method and assumptions are employed to analyse the dynamics of undulating fins, whose active deformation satisfies the kinematics model (4). More derivation and details are stated in [4]. The hydrodynamic forces and moment could be calculated according to the following dynamics model:

$$
\left[\begin{array}{c}
F_{X q} \\
F_{Y q} \\
F_{Z q} \\
M_{X q} \\
M_{Y q} \\
M_{Z q}
\end{array}\right]=\Gamma\left(f, U_{X}\right)\left[\begin{array}{c}
K_{1} \\
0 \\
0 \\
0 \\
K_{2} \sin \left(2 \pi f t+e_{0 q}^{M_{Y}}\right) \\
K_{3}+K_{4} \sin \left(4 \pi f t+e_{0 q}^{M_{Z}}\right)
\end{array}\right]
$$

where $\quad \Gamma\left(f, U_{X}\right)=-\operatorname{sign}\left(f+U_{X} / \lambda\right) \rho C s\left(f+U_{X} / \lambda\right)^{2} / 2$ is the kinematic coefficient that's independent of the coordinates variables $s$ and $l$. $U_{X}$ is the swimming velocity of the whole fin. The dynamic coefficients $K_{i}(i=1,2,3,4)$ are the functions of the design parameters, i.e., aspect ratio of the fin surface, the wavelength and the wave amplitude. Equation (7) indicates that the hydrodynamic forces and moments have relationships with both the undulation and the carrier motion. In this way, the design parameters could be described by $\Gamma\left(f, U_{X}\right)$ and $K_{i}(i=1,2,3,4)$.

\subsection{Simulation of the Dynamics Models}

According to the dynamic models, the parameters studies are further discussed. First of all, it is necessary to make $\Gamma\left(f, U_{X}\right)$ and $K_{i}(i=1,2,3,4)$ non-dimensional for catholicity. The dimension analysis of $\Gamma\left(f, U_{X}\right)$ and $K_{i}(i=1,2,3,4)$ are expressed as follows:

$$
\left[\Gamma\left(f, U_{X}\right)\right]=\left[F L^{-4}\right],\left[K_{1}\right]=\left[L^{4}\right], \quad\left[K_{i}\right]=\left[L^{5}\right],(i=2,3,4)
$$

Then $\Gamma\left(f, U_{X}\right)$ and $K_{i}(i=1,2,3,4)$ are non-dimensionalized:

$$
\begin{aligned}
\tilde{\Gamma}\left(f, U_{X}\right) & =\frac{\Gamma\left(f, U_{X}\right)}{-\operatorname{sign}(f) \rho C s f^{2} / 2} \\
\tilde{K}_{1} & =K_{1} / L^{4}, \tilde{K}_{i}=K_{i} / L^{5},(i=2,3,4)
\end{aligned}
$$

where $\tilde{\Gamma}\left(f, U_{X}\right)$ is dimensionless kinematic coefficient and $-U_{X} / \lambda f$ is dimensionless swimming velocity. Figure 11 shows the relation between $\tilde{\Gamma}\left(f, U_{X}\right)$ and $-U_{X} / \lambda f$. The force generated is kind of decided by $-U_{X} / \lambda f$, and Table 1 gives the basic principles. The thrust is generated as net force when $-U_{X} / \lambda f<1$, and resistance is generated when $-U_{X} / \lambda f>1$. In addition, $\Gamma\left(f, U_{X}\right)$ has an approximate second-degree polynomial relationship with the swimming velocity $U_{X}$ and wave frequency $f$. 


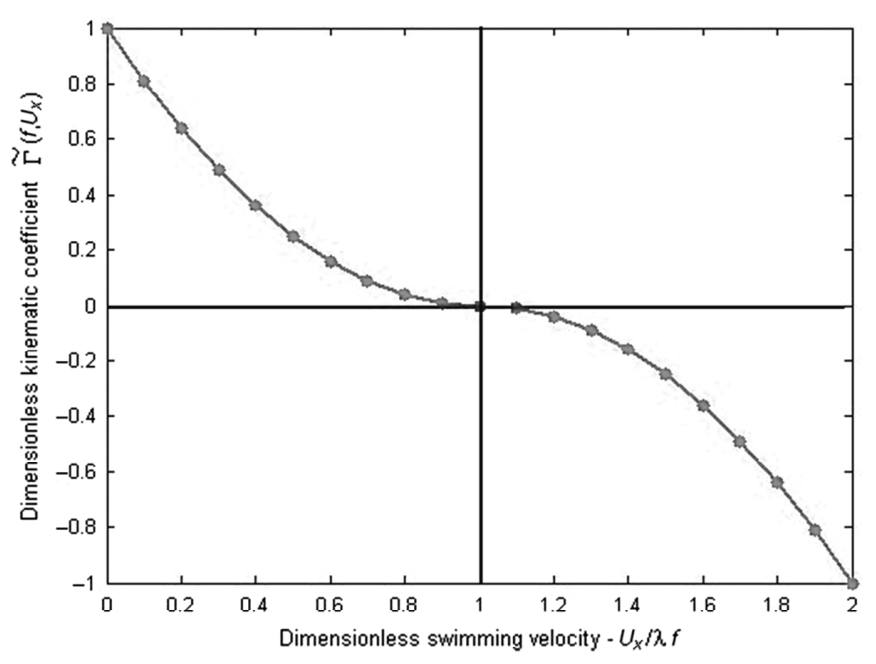

Figure 11. The relationship between $\tilde{\Gamma}\left(f, U_{X}\right)$ and $-U_{X} / \lambda f$ of dynamics simulation.

Table 1

Basic Principle of Thrust Generation

\begin{tabular}{|l|l|}
\hline Condition & Results \\
\hline$-U_{X} / \lambda f<1$ & Thrust generation \\
\hline$-U_{X} / \lambda f>1$ & Resistance generation \\
\hline$-U_{X} / \lambda f=1$ & Thrust is zero \\
\hline
\end{tabular}

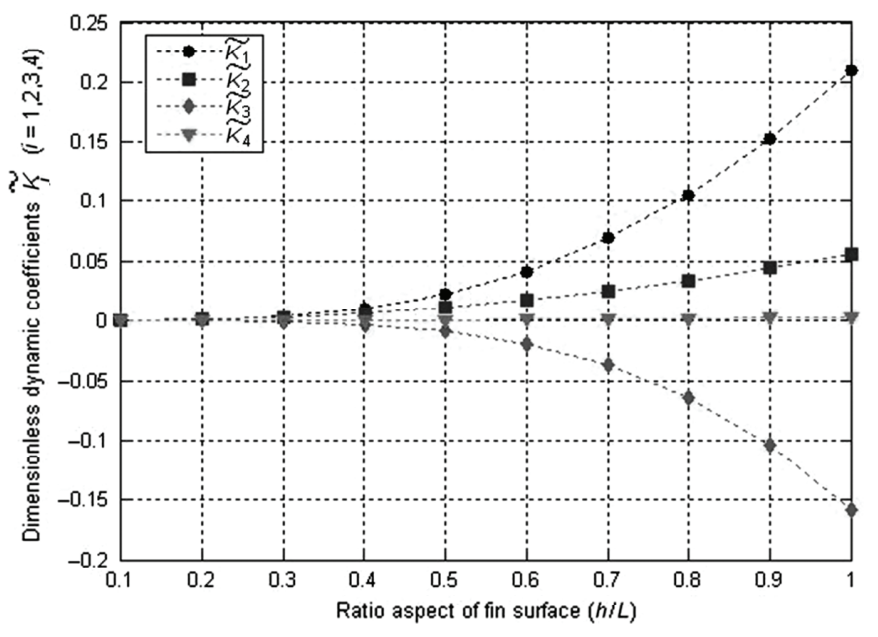

Figure 12. Effect of aspect ratio $h / L$ on $\tilde{K}_{i}$.

The parameters effect on $\tilde{K} i(i=1,2,3,4)$ is also discussed based the dynamics model, while the design parameters are taken into consideration, as shown in Figs. 12-14, which are the aspect ratio $h / L$, the maximum undulating angle $\theta_{\max }$ and the wave number $L / \lambda$, respectively.

There are steady and unsteady components contained in the forces and moments, as described in (7). It is preferred to keep the coefficients of steady components as a big value, such as $\tilde{K}_{1}$ and $\tilde{K}_{3}$, and the coefficients of periodic components as a small value, such as $\tilde{K}_{2}$ and $\tilde{K}_{4}$. Figures 12 and 13 show that $\tilde{K} i(i=1,2,3,4)$ have a square

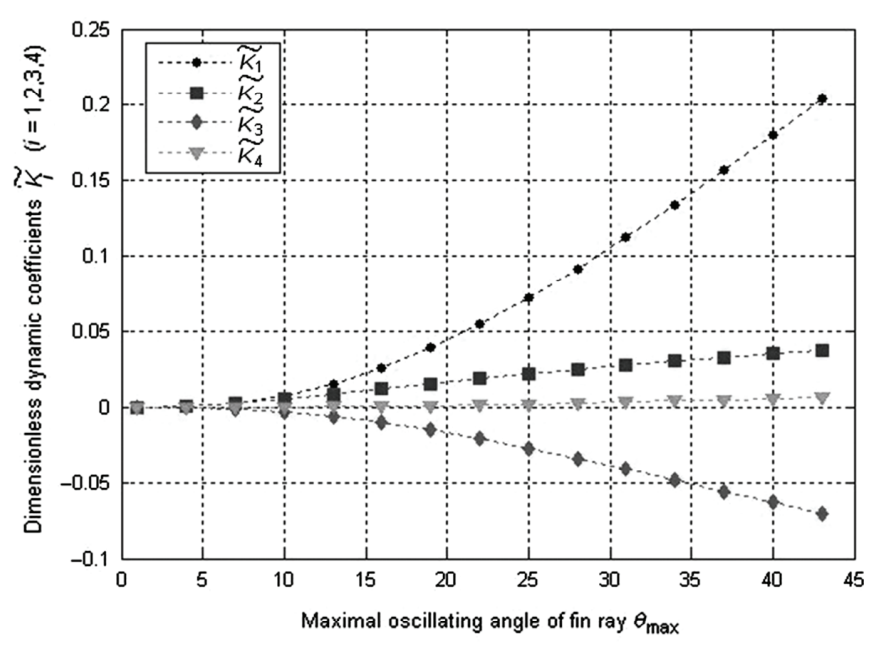

Figure 13. Effect of maximum undulating angle $\theta_{\max }$ on $\tilde{K}_{i}$.

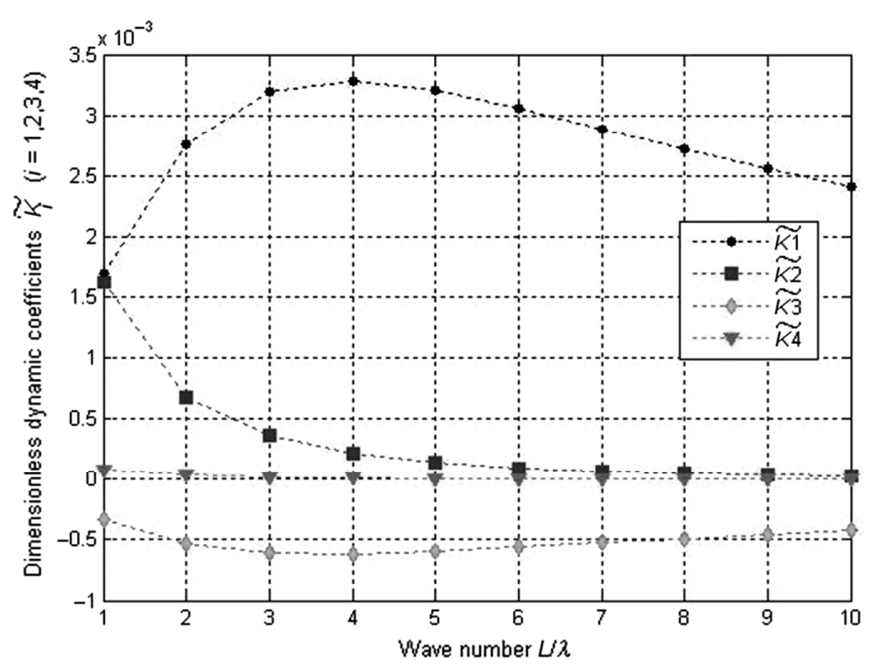

Figure 14. Effect of wave number $L / \lambda$ on $\tilde{K}_{i}$.

relationship with $h / L$ and $\theta_{\max }$. In the other hand, those design parameters are limited by the structural strength and driving capability of the actuating mechanism. The actuator and the fin surface are easy to be damaged or the undulating fin cannot achieve high frequency, if $h / L$ and $\theta_{\max }$ are too big. The coefficients $\tilde{K}_{2}$ and $\tilde{K}_{4}$ decrease monotonically as the wave number $L / \lambda$ increases. There is an optimal $L / \lambda$ which makes $\tilde{K}_{1}$ and $\tilde{K}_{3}$ achieve the extremum, as shown in Fig. 14. The coefficient $\tilde{K}_{4}$ is small enough to be negligible relative to other coefficients, which is also insensitive to $h / L, \theta_{\max }$ and $L / \lambda$. Therefore it could be ignored in practice. Those relationships obtained by dynamics analysis would provide valued references for the bionic fin design.

\section{Initial Experiment of Bionic Undulating Fin}

The independently driven bionic fin is used for the kinematics and dynamics analysis, and verifies the proposed dynamics model. The thrust and pitching moment are 


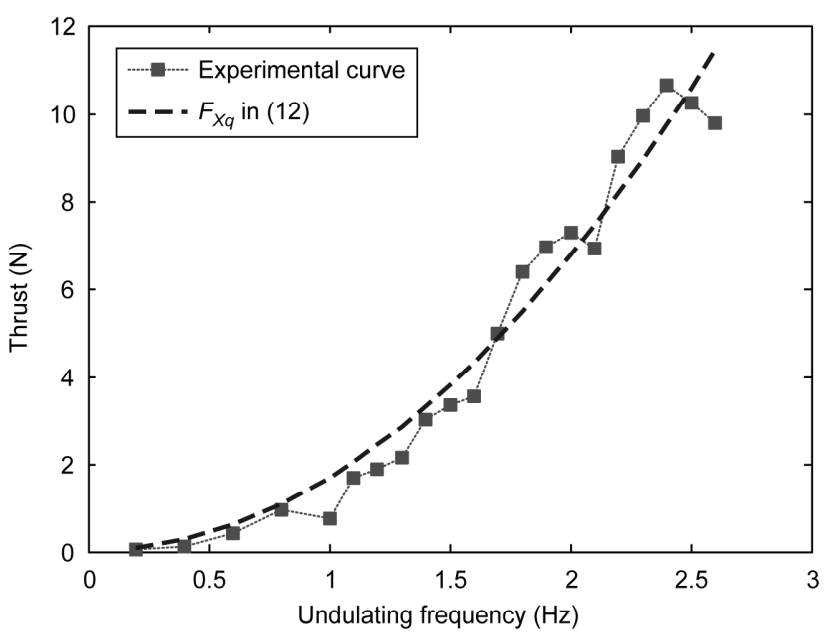

Figure 15. Experimental and theoretical comparison of the relationship between thrust and undulating frequency.

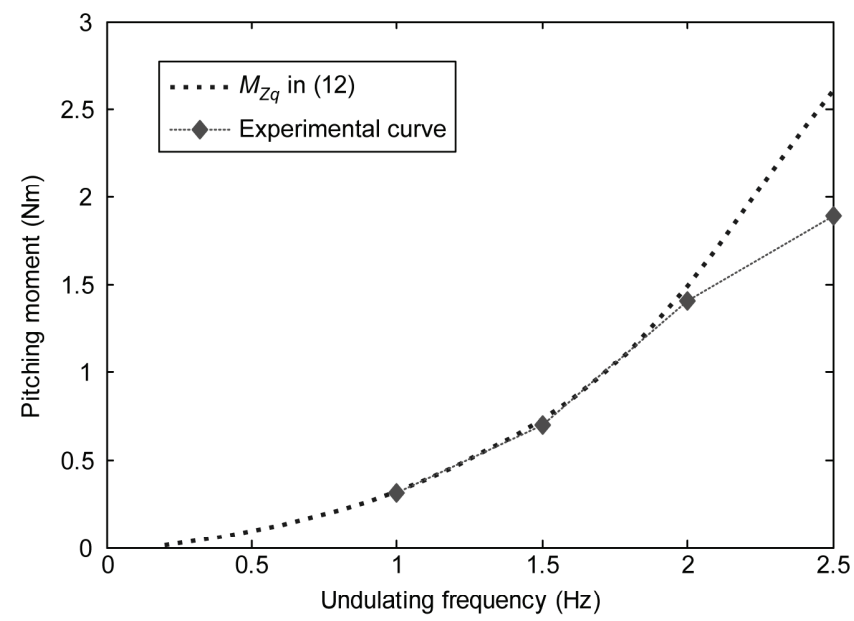

Figure 16. Experimental and theoretical comparison of relationship between pitching moment and the undulating frequency.

experimentally measured under the condition of $U_{X}=0$. The base of the bionic fin is fixed in position. The trust is measured by dynamometers directly. The measuring results of dynamometers are transferred to the moment through multiplying the corresponding arm of force. The fin length is $L=0.6 \mathrm{~m}$ and the maximal undulating angle is $\theta_{\max }=15^{\circ}$ in the experiment. The wavelength is $\lambda=0.6 \mathrm{~m}$, thus the wave number is $n=1$. The width of fin surface is $h=0.15 \mathrm{~m}$, thus the aspect ratio is $h / L=0.25$. The density of fluid is $\rho=10^{3} \mathrm{~kg} / \mathrm{m}^{3}$.

Figure 15 shows the thrust against undulating frequency which varies from 0.2 to $2.6 \mathrm{~Hz}$. The experimental result is compared with the theoretical result. In addition, drag coefficient of the surface element $C_{s}$ in (7) could be further identified, which is unknown in the theoretical model. Based on the experimental data, $C_{s}$ is identified to be 15.4 using the least-square method. The theoretical and experimental results of the pitching moment are shown in Fig. 16. The theoretical curves are substantial agreement with the experimental curves. The thrust and
Table 2

Velocity Test Results with Different Undulating

Parameters

\begin{tabular}{|c|c|c|}
\hline \multirow{2}{*}{$\begin{array}{c}\text { Undulating } \\
\text { Frequency }\end{array}$} & \multicolumn{2}{|c|}{ Velocity (m/s) } \\
\cline { 2 - 3 } & $\begin{array}{c}\text { Aspect Ratio: 0.25 } \\
\text { Length of Fin Ray: } \\
0.15 \mathrm{~m}\end{array}$ & $\begin{array}{c}\text { Aspect Ratio: 0.167 } \\
\text { Length of Fin Ray: } \\
0.10 \mathrm{~m}\end{array}$ \\
\hline 2.0 & 0.284 & - \\
\hline 2.5 & 0.334 & 0.195 \\
\hline 3.0 & 0.362 & 0.222 \\
\hline 3.5 & 0.400 & 0.256 \\
\hline 4.0 & 0.424 & 0.283 \\
\hline 4.5 & 0.416 & 0.305 \\
\hline
\end{tabular}

pitching moment both have square relationships with the frequency. But the thrust or pitching moment won't increase any more after the frequency is bigger than $2.3 \mathrm{~Hz}$. This phenomenon is probably because that the undulating fin could not reach the desired frequency when the driving system enters into its saturation region.

The relationships between wave frequency and hydrodynamic thrust and pitching moment are tested while $U_{X}$ is zero. We further test the relationship between the steady zero thrust velocity and undulating parameters. Zero thrust velocity means that the thrust and drag results balance, when the net force is nearly zero and the underwater device swims in steady velocity along the axis direction. The aspect ratio of fin ray is set as 0.25 and 0.167 . The velocity test results under different undulating frequency is given in Table 2. The zero thrust velocity and wave frequency show approximately linear proportional relationship. Computational fluid dynamics (CFD) analysis showed that the relationship curve should be nearly quadratic function [28]. But the experimental results show more closely a linear relationship because the torque and response velocity of the motor are limited. When the load exceeds limit values, the desired undulating frequency could not be reached, and swimming velocity could not increase anymore. Besides, bigger aspect ratio results in greater velocity under our experimental set-up conditions.

\section{Conclusion}

In this paper, both fixed-waveform and independently driven bionic propulsors are designed which imitate the undulating fish fin. The kinematics model is established to describe undulating motion, and the simulation result shows good performance. The dynamics of the bionic undulating fin is theoretical analysed using the fluid drag model. The effects on dynamics of geometric parameters, undulating parameters as well as the swimming velocity are also studied by simulation. The experimental results show that the proposed kinematics and dynamics models 
are effective for the characteristic investigation of the undulating motion. The kinematics and dynamics models will further provide guidance for the mechanical structure and control system development. The potential flow theory and the hydrodynamic experiment will be further exploited to establish the more accurate model.

\section{References}

[1] P.W. Webb, Form and function in fish swimming, Scientific American, 251(1), 1984, 58-68.

[2] M. Sfakiotakis, D.M. Lane, and J.B.C. Davies, Review of fish swimming modes for aquatic locomotion, IEEE Journal of Oceanic Engineering, 24(2), 1999, 237-252.

[3] H.W. Lissmann and K.E. Machin, The mechanism of object location in Gymnarchus niloticus and similar fish, Journal of Experimental Biology, 35(2), 1958, 451-486.

[4] H. Xie and L. Shen, Dynamic analysis on the bionic propulsor imitating undulating fin of aquatic animals, IEEE International Conf on Robotics and Biomimetics, Sanya, China, 2007, 268-273.

[5] C. Zhou, Z. Cao, S. Wang, and T. Min. The design, modelling and implementation of a miniature biomimetic robotic fish, International Journal of Robotics 85 Automation, 25(3), 2010, $210-216$.

[6] R. Hooshmand, A.A. Nasiri, and M. Ataei, Trajectory angle control of fish-like robot motion by using fuzzy-PID controller, International Journal of Robotics \& Automation, 27(2), 2012, $163-176$.

[7] S. Subramanian, T. George, and A. Thondiyath, Real-time obstacle avoidance for an underactuated flat-fish type autonomous underwater vehicle in 3D space, International Journal of Robotics \& Automation, 29(4), 2014, 424-431.

[8] L. Shen and G. Wang, The progress and analysis of the research on the underwater biomimetic propulsor employing the longfin undulations, Journal of National University of Defense Technology, 27(4), 2005, 96-100 (in Chinese).

[9] M. Sfakiotakis, D.M. Laue, and B.C. Davies, An experimental undulating-fin device using the parallel bellows actuator, Proc. 2001 IEEE International Conf. on Robotics \& Automation, Seoul, Korea, 2001, 2356-2362.

[10] M.A. MacIver, E. Fontaine, and J. W. Burdick, Designing future underwater vehicles: Principles and mechanisms of the weakly electric fish, IEEE Journal of Oceanic Engineering, 29(3), 2004, 651-659.

[11] M. Epstein, J.E. Colgate, and M.A. MacIver, A biologically inspired robotic ribbon fins, Proc. IEEE/RSJ International Conf. on Intelligent Robots and Systems, Workshop on Morphology, Control, and Passive Dynamics, Edmonton, Alberta, Canada, 2005.

[12] K.H. Low and A. Willy, Biomimetic motion planning of an undulating robotic fish, Journal of Vibration and Control, 12(12), 2006, 1337-1359.

[13] K.H. Low, Design, development and locomotion control of bio-fish robot with undulating anal fins, International Journal of Robotics and Automation, 22(1), 2007, 88-99.

[14] K.H. Low, C. Zhou, and Y. Zhong, Gait planning for steady swimming control of biomimetic fish robots, Advanced Robotics, 23(7-8), 2009, 805-829.

[15] K.H. Low and C.W. Chong, Parametric study of the swimming performance of a fish robot propelled by a flexible caudal fin, Bioinspiration \& Biomimetics, 5(4), 2010, 046002.

[16] Y. Toda, S. Hieda, and T. Sugiguchi, Laminar flow computation around a plate with two undulating side fins, Journal of Kansai Society of Naval Architects, (237), 2002, 71-78.

[17] Y. Toda, H. Ikedab, and N Sogihara, The motion of a fish-like under-water vehicle with two undulating side fins, The Third International Symposium on Aero Aqua Bio-Mechanisms, Ginowan, Okinawa, Japan, 2006.

[18] H. Xie, L. Shen, and T. Hu, Movement curved surface modeling and simulation of long flexible fin of Gymnarchus niloticus, Journal of National University of Defense Technology, 27(5), 2005, 62-66 (in Chinese).
[19] Y. Zhang, L. Jia, S. Zhang, J. Yang, et al., Computational research on modular undulating fin for biorobotic underwater propulsor, Journal of Bionic Engineering, 4(1), 2007, 25-32.

[20] R. Bandyopadhyay, Trends in biorobotic autonomous undersea vehicles, IEEE Journal of Oceanic Engineering, 30(1), 2005, 109-139.

[21] F. Boyer and P. Mathieu, Multibody system dynamics for bio-inspired locomotion: From geometric structures to computational aspects, Bioinspiration \& Biomimetics, 10(2), 2015, 025007.

[22] J.E. Colgate and K.M. Lynch, Mechanics and control of swimming: A review, IEEE Journal of Oceanic Engineering, 29(3), 2004, 660-673.

[23] R.W. Blake, Fish functional design and swimming performance, Journal of Fish Biology, 65(5), 2004, 1193-1222.

[24] M.J. Lighthill and R.W. Blake, Biofluid dynamics of Balistiform and Ggymnotiform locomotion. Part 1. Biological background, and analysis by elongated-body theory, Journal of Fluid Mechanism, 212, 1990, 183-207.

[25] B. Tong, L. Zhuang, and J. Cheng, Study on hydrodynamics of propulsion by fish's undulatory motion, Mechanics in Engineering, 13(3), 1991, 17-26 (in Chinese).

[26] B. Tong, Propulsion mechanism of fish's undulatory swimming, Mechanics in Engineering, 22(3), 2000, 69-74 (in Chinese).

[27] G.V. Lauder, E.J. Anderson, J. Tangorra, and P. G. A. Madden, Fish biorobotics: Kinematics and hydrodynamics of self-propulsion, Journal of Experimental Biology, 210(16), 2007, 2767-2780.

[28] H. Zhou, T. Hu, H. Xie, D. Zhang, et al., Computational hydrodynamics and statistical modeling on biologically inspired undulating robotic fins: A two-dimensional study, Journal of Bionic Engineering, 7(1), 2010, 66-76.

\section{Biographies}

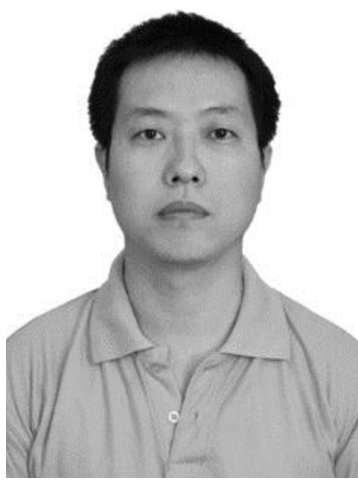

Haibin Xie received the B.E., M.S. and Ph.D. degrees in control science and engineering from the National University of Defense Technology (NUDT), Changsha, China, in 1999, 2002, and 2006, respectively. In 2006, he joined the College of Mechatronics and Automation, NUDT, where he is currently an Associate Professor. His research interests include biomimetic robots, artificial intelligence, computational intelligence and unmanned systems. He is a founding member of International Society of Bionic Engineering and senior member of Chinese Association of Automation.

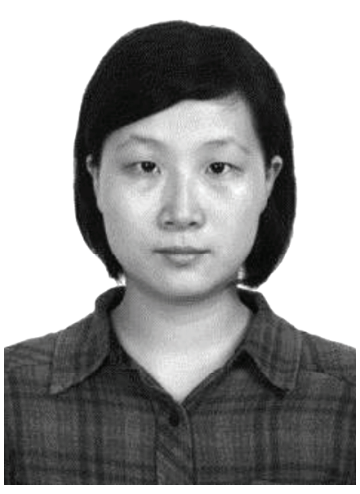

Han Zhou received the Ph.D. degree in control science and engineering from the National University of Defense Technology (NUDT), Changsha, China, in 2015. From November 2011 to November 2012, she was with University of Essex, UK, as a visiting scientist. Since 2015, she has been with the Faculty of College of Mechatronics and Automation, NUDT. Her research interests include biomimetic robotics, dynamics analysis and learning control. 


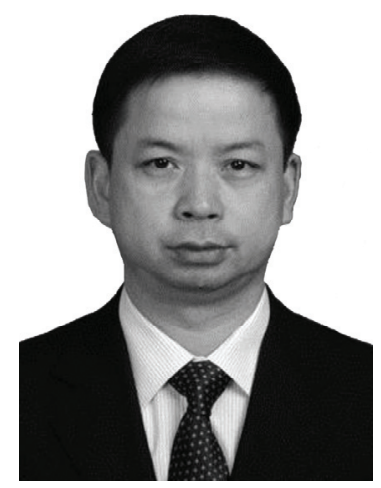

Lincheng Shen received the B.E., M.S., and Ph.D. degrees in automatic control from the National University of Defense Technology (NUDT), Changsha, China, in 1986,1989 , and 1994, respectively. In 1989, he joined the Department of Automatic Control, NUDT, where he is currently a Full Professor and serves as the Dean of the College of Mechatronics and Automation. His research interests include mission planning, autonomous and cooperative control for unmanned systems, biomimetic robotics and intelligent control.

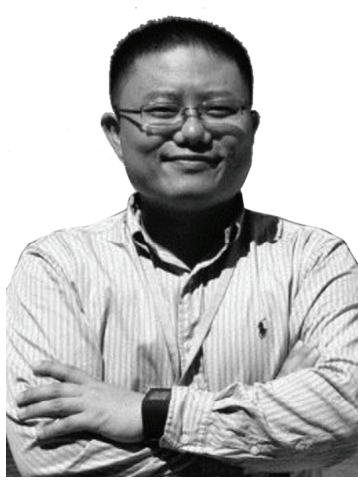

Dong Yin has been a faculty of College of Mechatronic Engineering and Automation in National University of Defense Technology since 2011. He got his bachelor degree, master's degree and doctor degree in Automation College of Northwestern Polytechnical University at 2004, 2007 and 2011, respectively, and had been a visiting scholar in department of ECE at University of Massachusetts from 2008 to 2010. Now his research interests include network virtualization, network control and information security. 